\title{
Effects of Astragaloside on Biological Characteristics of Non-Small Cell Lung Cancer Cells via the Phosphatidylinositol 3-Kinase/Protein Kinase B/ Mechanistic Target of Rapamycin Pathway
}

\author{
YUXIA LIU, YUE ZHAO ${ }^{1}$, Z. PENG, S. LI, AISHUAI WANG²AND X. ZHENG ${ }^{3 *}$ \\ Department of Respiratory Medicine, ${ }^{1}$ Department of VIP Care, Second Affiliated Hospital of Shandong University of \\ Traditional Chinese Medicine, Jinan 250011, ${ }^{2}$ Department of Traditional Chinese Medicine, Shandong Provincial Third \\ Hospital, Jinan 250031, ${ }^{3}$ Qingdao Hospital of Traditional Chinese Medicine, Qingdao 266000, Shandong Province, China
}

Liu et al.: Effects of Astragaloside on Lung Cancer Cells

\begin{abstract}
To evaluate the effects of astragaloside on the proliferation, invasion, migration and apoptosis of nonsmall cell lung cancer A549 cells and phosphatidylinositol 3-kinase/protein kinase B/mechanistic target of rapamycin pathway. After A549 cells were treated with 0, 10, 20, 40, 60, 80 and $100 \mu \mathrm{mol} / \mathrm{l}$ astragaloside for $24 \mathrm{~h}$, the survival rate was measured by methyl thiazolyl tetrazolium assay and the half maximal inhibitory concentration was calculated. Astragaloside at half maximal inhibitory concentration was added. Cell invasion, migration and apoptosis were detected by transwell assay, wound healing assay and flow cytometry, respectively. The effects of astragaloside on the expressions of phosphatidylinositol 3-kinase/protein kinase $B$ /mechanistic target of rapamycin pathway-related proteins were detected by western blotting. After different concentrations of astragaloside were added, cell proliferation declined with increasing concentration. Half maximal inhibitory concentration against A549 cell proliferation was 32.2 $\mu \mathrm{mol} / \mathrm{l}$. Compared with blank control group; cell proliferation; invasion and migration were significantly weakened, while apoptosis was enhanced in astragaloside group. The expressions of phosphorylation of phosphatidylinositol 3-kinase, phosphorylation of protein kinase B and phosphorylation of mechanistic target of rapamycin proteins in astragaloside group were significantly lower than those in blank control group. Astragaloside inhibits the proliferation, invasion and migration, and promotes the apoptosis of nonsmall cell lung cancer A549 cells dose-dependently. Inhibiting phosphorylation of phosphatidylinositol 3-kinase, phosphorylation of protein kinase $B$ and phosphorylation of mechanistic target of rapamycin expressions in the phosphatidylinositol 3-kinase/protein kinase B/mechanistic target of rapamycin pathway may be one of the mechanisms of astragaloside.
\end{abstract}

Key words: Astragaloside, non-small cell lung cancer, proliferation, migration, apoptosis

Lung cancer is a solid malignancy, and its morbidity and mortality rates rank among the top of malignant tumors, which is considered as a serious threat to human life and health around the world ${ }^{[1,2]}$. Based on pathological characteristics, lung cancer is classified into non-small cell lung cancer (NSCLC) and SCLC. As a major subtype of lung cancer, NSCLC accounts for about $85 \%$ of lung cancer ${ }^{[3-6]}$. With the deepening of basic research and advances in technology, a variety of treatment means have been gradually applied for NSCLC, including surgery, chemotherapy, immunotherapy, radiotherapy and targeted therapy ${ }^{[7,8]}$. Currently, NSCLC is not sensitive to chemotherapy and

*Address for correspondence E-mail: zhengxin66999@163.com is prone to drug resistance, so the $5 \mathrm{y}$ survival rate of patients is only $10-15 \%{ }^{[9,10]}$. Therefore, it is of important practical significance to develop new drugs and clarify the mechanism of action for the treatment of NSCLC. Astragaloside (AS) is the main active ingredient extracted from Radix Astragali and it has been proved to play an important role in anti-inflammation, antiThis is an open access article distributed under the terms of the Creative
Commons Attribution-NonCommercial-ShareAlike 3.0 License, which
allows others to remix, tweak, and build upon the work non-commercially,
as long as the author is credited and the new creations are licensed under
the identical terms

Accepted 22 August 2021

Revised 28 January 2021

Received 10 November 2019 Indian J Pharm Sci 2021;83(4):865-870 
oxidation, blood glucose reduction and improvement of cardiovascular diseases ${ }^{[11-13]}$. In recent years, the potential value of traditional Chinese medicine in the anti-tumor field has been paid increasingly more attention to and the research on anti-tumor effect of traditional Chinese medicine is gradually being conducted on a large scale. Studies have demonstrated that AS can effectively inhibit the development of various tumor cells, such as liver cancer, human neuroblastoma and gastric cancer cells, whose antitumor effect is exerted mainly by enhancing the in vivo immune response inhibiting lung cancer ${ }^{[14-16]}$. It has been found that AS can effectively restrain the development of lung cancer cells ${ }^{[17,18]}$, but the anticancer activity of AS at different concentrations and its exact mechanism remain to be further clarified. In the present study, NSCLC A549 cells were used as the in vitro cell model, the regulatory effects of $\mathrm{AS}$ in different doses on the proliferation, invasion and apoptosis of cancer cells were studied, and its potential mechanism of action was explored, so as to offer a new theoretical basis and a potential target to the clinical treatment of NSCLC. Human NSCLC A549 cells were purchased from the Shanghai Cell Bank of the Chinese Academy of Sciences, and cultured in vitro in Roswell Park Memorial Institute (RPMI) 1640 medium supplemented with $10 \%$ fetal bovine serum (FBS) and $1 \%$ penicillin-streptomycin double antibody in an incubator with $5 \% \mathrm{CO}_{2}$ at $37^{\circ}$. AS (Shanghai Yuanye Biological Co., Ltd., China) was dissolved with dimethyl sulfoxide (DMSO, Sigma, USA), prepared into $10 \mathrm{mmol} / 1$ original drug stock solution, and stored in a refrigerator at $4^{\circ}$ for later use. Phenylmethylsulfonyl fluoride, protease inhibitor mixture, protein phosphatase inhibitor mixture and high-efficiency Radioimmunoprecipitation assay (RIPA) lysis buffer (Beyotime Institute of Biotechnology, China), goat anti-mouse phosphorylation of protein kinase B (p-Akt) and Akt monoclonal primary antibodies and rabbit antimouse phosphorylation of mechanistic target of rapamycin (p-mTOR) and mTOR monoclonal antibodies (Santa Cruz, USA), methyl thiazolyl tetrazolium (MTT) kits (Beijing Solarbio Science \& Technology Co., Ltd., China), Annexin V-FITC (Beyotime Institute of Biotechnology, China), bicinchoninic acid (BCA) protein concentration assay kits (Beyotime Institute of Biotechnology, China), multifunctional microplate reader (BioTek, USA), flow cytometer (BD, USA), and quantitative polymerase chain reaction (qPCR) system (Applied Biosystems, USA) were used. A549 cells in the logarithmic growth phase were digested with trypsin and prepared into cell suspension with RPMI-1640 medium containing $10 \%$ FBS, with a cell density of $1 \times 10^{5}$ cells $/ \mathrm{ml}$. The cell suspension was pipetted into a 96-well plate $(200 \mu \mathrm{l} /$ well) and cultured in an incubator with $5 \% \mathrm{CO}_{2}$ at $37^{\circ}$ for $24 \mathrm{~h}$. After the cells adhered to the wall, the medium was replaced with $0,10,20,40,60,80$ and 100 $\mu \mathrm{mol} / 1 \mathrm{AS}$, followed by culture for another $24 \mathrm{~h}$. After treatment, the cells were washed with phosphate buffered saline (PBS) and cultured with $100 \mu \mathrm{l}$ of medium and $20 \mu \mathrm{l}$ of MTT $(5 \mathrm{mg} / \mathrm{ml})$ for $4 \mathrm{~h}$ and then the supernatant was discarded. Finally, $150 \mu 1$ of DMSO was added into each well, the 96-well plate was vibrated on a horizontal shaker for $10 \mathrm{~min}$ and the optical density (OD) was measured at $490 \mathrm{~nm}$ using a microplate reader. The cell growth inhibition rate was calculated using the following formula: growth inhibition rate $=(1-$ mean OD in AS group/mean OD in control group) $\times 100 \%$. The half maximal inhibitory concentration $\left(\mathrm{IC}_{50}\right)$ of AS on A549 cell proliferation at $24 \mathrm{~h}$ was calculated. Matrigel diluted with serum-free medium was added into the upper transwell chamber, and air dried at $37^{\circ} .200 \mu$ of cell suspension was added into the upper chamber, while $500 \mu$ of RPMI-1640 medium containing serum was added into the lower chamber, followed by treatment with $\mathrm{AS}$ at an $\mathrm{IC}_{50}$ under $5 \% \mathrm{CO}_{2}$ and $37^{\circ}$ for $24 \mathrm{~h}$. The blank control group was set up (an equal dose of DMSO solution). Matrigel and cells in the upper chamber were wiped off with cotton swabs, fixed with paraformaldehyde and stained, followed by observation and photography under an optical microscope in 5 randomly selected fields of view for each sample. A549 cells in good growth conditions were collected, digested with trypsin and centrifuged. $2 \mathrm{ml}$ of cells were inoculated into a 6-well plate (about $4 \times 10^{5}$ cells/well) and cultured until the cell density reached $80-90 \%$. Then the cell monolayer was quickly scratched straight with $10 \mu$ of sterile tips and the cells were washed with PBS 3 times to remove cell debris. The original medium containing $10 \%$ serum was replaced with serum-free medium, AS at an $\mathrm{IC}_{50}$ was added and the blank control group was set up (an equal dose of DMSO solution). The wound healing was observed under the microscope at $0 \mathrm{~h}$ and $24 \mathrm{~h}$ after AS was added and photographed in 4 different fields of view in each group, based on which the cell recovery status was detected. The wound healing rate was calculated using the following formula: wound healing rate $=$ (wound area immediately after scratchwound area at $24 \mathrm{~h}$ after scratch)/wound area immediately after scratch $\times 100 \%$. A549 cells in 
logarithmic growth phase were collected, prepared into single cell suspension and inoculated into the medium with $\mathrm{AS}$ at an $\mathrm{IC}_{50}$. The blank control group was set up (an equal dose of DMSO solution). After routine culture for $24 \mathrm{~h}$, the cells were collected, washed twice with pre-cooled PBS and diluted until the cell concentration was $1 \times 10^{5}$ cells $/ \mathrm{ml}$. Then $490 \mu \mathrm{l}$ of cell suspension was placed into the flow tube, added with $5 \mu$ of Annexin V-FITC and incubated at room temperature for $10 \mathrm{~min}$. After $5 \mu \mathrm{l}$ of propidium iodide (PI) was added, flow cytometry was immediately performed. A549 cells in logarithmic growth phase were inoculated into a 6-well plate (about $2 \times 10^{5}$ cells/well) and treated with AS at an $\mathrm{IC}_{50}$ (AS group) and the blank control group was set up. After $24 \mathrm{~h}$, the cells were lysed with RIPA lysis buffer to extract the protein and the total protein was quantified by the bicinchoninic acid (BCA) method. $60 \mathrm{ng}$ of total protein in each group was loaded, subjected to $10 \%$ sodium dodecyl sulphatepolyacrylamide gel electrophoresis (SDS-PAGE), and transferred onto a polyvinylidene fluoride (PVDF) membrane. Then the PVDF membrane was washed with mixture of tris-buffered saline (TBS) and tween 20 (TBST), blocked with $5 \%$ horse serum at room temperature for $30 \mathrm{~min}$, and incubated with specific primary antibodies diluted with TBST at $4^{\circ}$ overnight. Then the membrane was washed with TBST 2-3 times (10 $\mathrm{min} /$ time) and incubated with the secondary antibodies at room temperature for $2 \mathrm{~h}$, followed by auto-fluorescence development using enhanced chemiluminescence (ECL) kits. The protein electrophoresis bands were recorded using a gel imaging system and its gray value was measured by ImageJ software. The semi-quantitative value of the target protein was expressed as the ratio of the gray value of target protein to that of internal reference. Statistical package for the social sciences (SPSS) 23.0 software was used for statistical analysis. Measurement data were expressed as mean \pm standard deviation and intergroup comparison was conducted by t test. $\mathrm{p}<0.05$ was considered statistically significant. After different concentrations of AS were added, the proliferative activity of A549 cells gradually declined as the concentration of AS was increased. At $24 \mathrm{~h}$ after treatment with $0,10,20,40,60,80$ and $100 \mu \mathrm{mol} / 1 \mathrm{AS}$, the survival rate of A549 cells was $(100.28 \pm 1.34) \%$, $(90.11 \pm 2.98) \%,(72.78 \pm 2.43) \%,(35.67 \pm 3.18) \%$, $(24.21 \pm 1.53) \%,(13.22 \pm 1.12) \%$ and $(6.84 \pm 1.02) \%$, respectively. According to the cell proliferative activity curve, the $\mathrm{IC}_{50}$ of AS on A549 cell proliferation at $24 \mathrm{~h}$ after treatment was $32.2 \mu \mathrm{mol} / 1$ (fig. 1). The number of invading cells in AS group was significantly smaller than that in control group [(103 \pm 15$)$ vs. $(354 \pm 21)]$ $(\mathrm{p}<0.001)$, suggesting that AS can greatly inhibit the invasion of A549 cells (fig. 2). The cell migration rate at $24 \mathrm{~h}$ in AS group was significantly lower than that in control group $[(25.47 \pm 2.38) \%$ vs. $(73.24 \pm 4.39) \%]$ $(\mathrm{p}<0.001)$, indicating that AS can significantly suppress the migration of A549 cells (fig. 3). The apoptosis rate at $24 \mathrm{~h}$ in AS group was significantly higher than that in control group $[(25.67 \pm 1.86) \%$ vs. $(11.78 \pm 2.27) \%$ ] $(p<0.001)$, indicating that AS can obviously enhance the apoptosis of A549 cells (fig. 4). The results of western blotting showed that AS group had significantly lower expressions of p-PI3K, p-Akt and p-mTOR proteins than control group. It can be seen that AS can

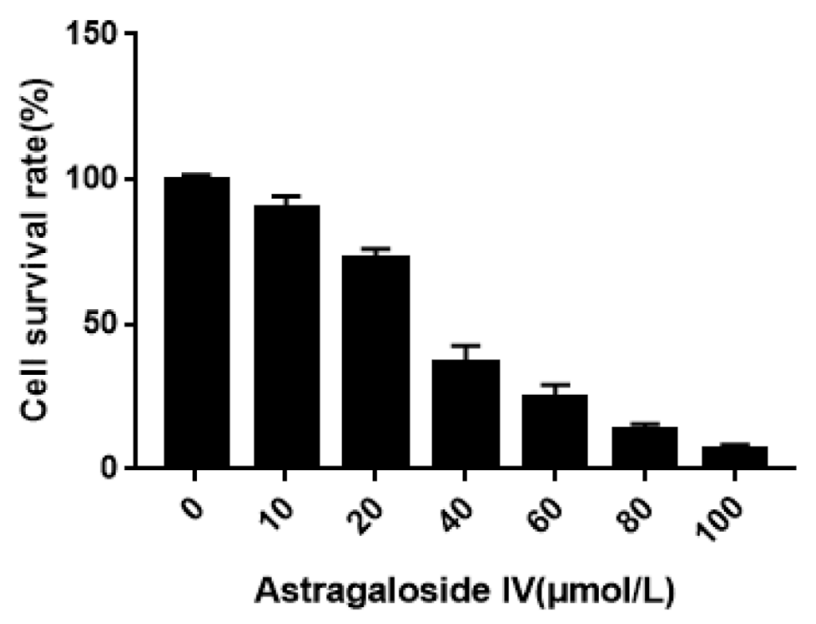

Fig. 1: Survival rate of A549 cells $24 \mathrm{~h}$ after addition of AS at different concentrations

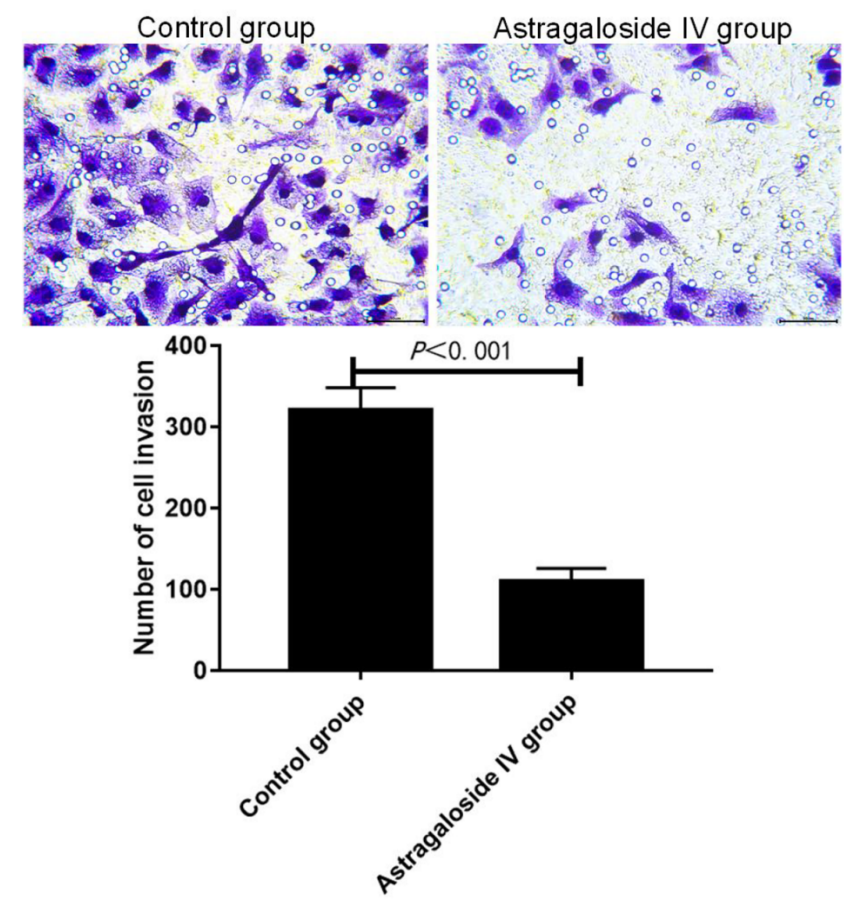

Fig. 2: Effect of AS on invasion ability of A549 cells 

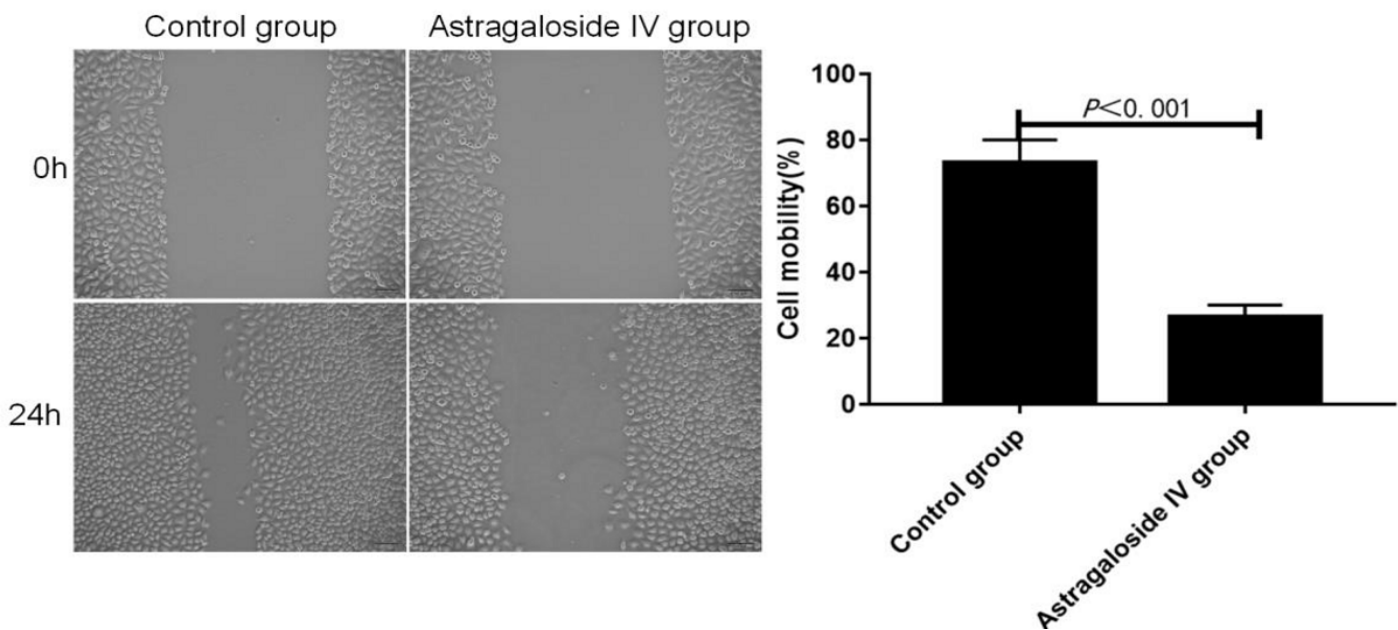

Fig. 3: Effect of AS on migration ability of A549 cells
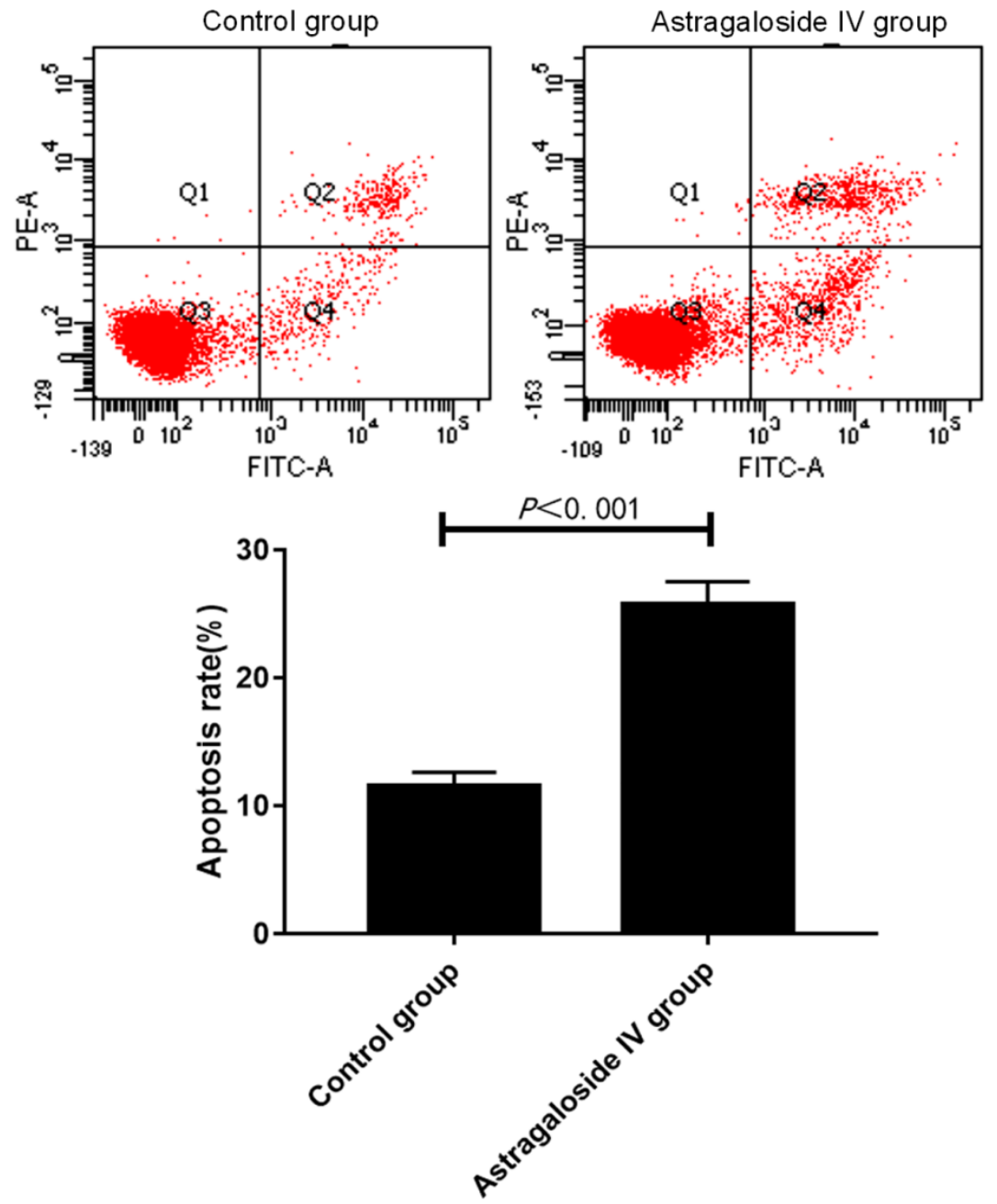

Fig. 4: Effect of AS on A549 cell apoptosis

markedly inhibit the expressions of phosphorylation of phosphatidylinositol 3-kinase (p-PI3K), p-Akt and p-mTOR proteins, and the effects of AS of inhibiting cell proliferation, migration and invasion, and promoting apoptosis may be achieved by regulating the $\mathrm{PI} 3 \mathrm{~K} /$ Akt/mTOR signaling pathway (fig. 5). Lung cancer is one of the globally recognized malignancies with the highest morbidity and mortality rates. The $5 \mathrm{y}$ survival rate of patients with NSCLC remains low, so it is of important practical significance to develop novel anti-tumor drugs for the treatment of NSCLC. AS, the main active ingredient extracted from Radix Astragali, has been proved to play an important role in antiinflammation, anti-oxidation, blood glucose reduction 


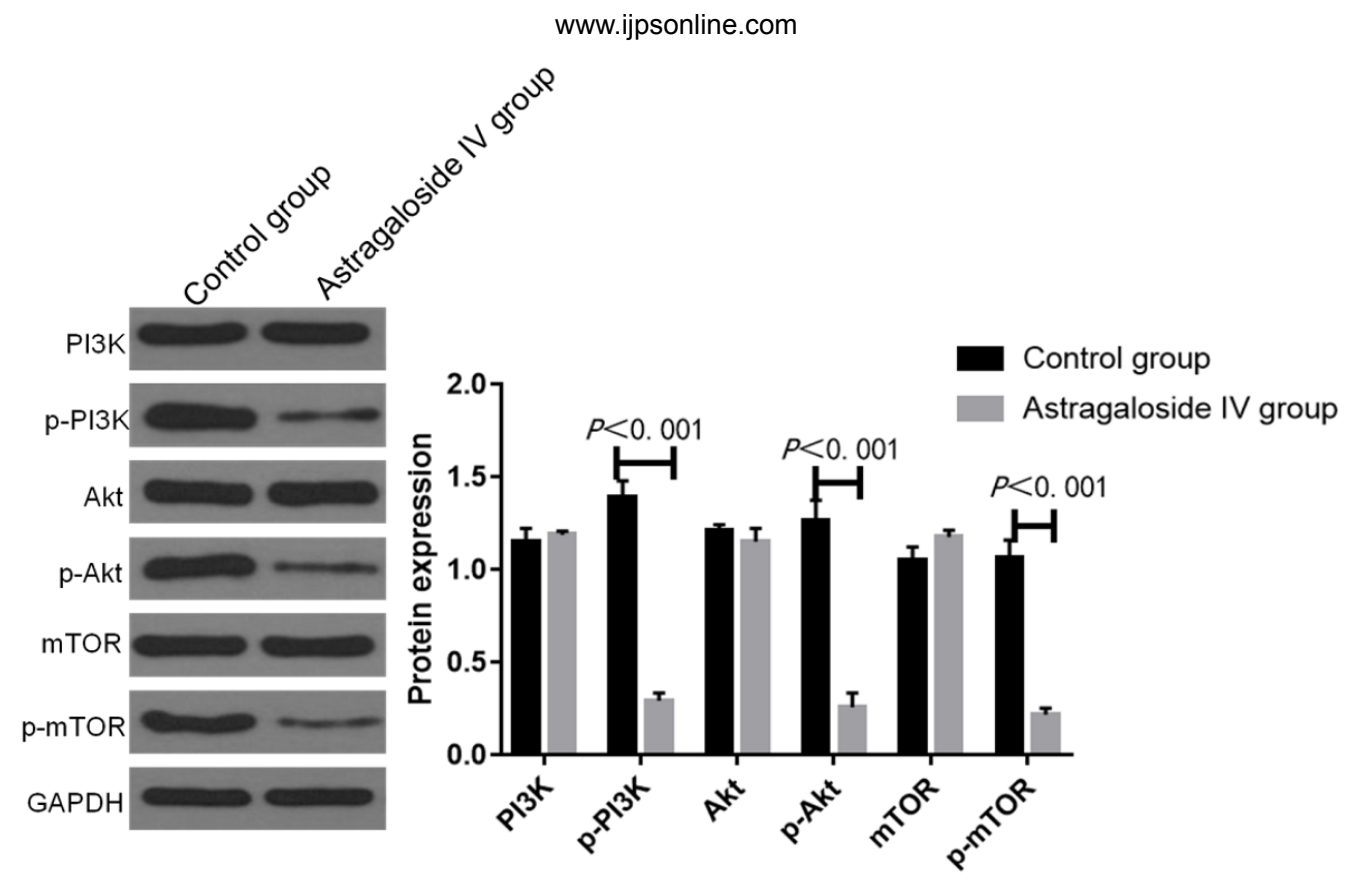

Fig. 5: Effects of AS on expressions of PI3K/Akt/mTOR signaling pathway-related proteins in lung cancer cells

and improvement of cardiovascular diseases ${ }^{[11,12]}$. A recent study ${ }^{[18]}$ has shown that AS also exerts a significant inhibitory effect on tumors and it may work through the Wnt/beta-catenin signaling pathway ${ }^{[19]}$. AS inhibits the in vitro proliferation, migration and invasion of ovarian cancer SKOV3 cells, which may be related to its down-regulation on the expressions of matrix metalloproteinase-2 (MMP-2) and MMP-9 proteins ${ }^{[20]}$. At present, the regulatory effect of AS on NSCLC cells is still unclear and its mechanism of action remains to be explored. In the present study, the effects of AS in different doses on the proliferative activity of A549 cells were analyzed by MTT assay first and it was determined that the $\mathrm{IC}_{50}$ of AS at $24 \mathrm{~h}$ was $32.2 \mu \mathrm{mol} / 1$, confirming that AS suppresses the A549 cell proliferation in a dose-dependent manner. Then the invasion, migration and apoptosis of A549 cells were detected by transwell assay, wound healing assay and flow cytometry, respectively. It was found that AS could greatly inhibit invasion and migration and enhance apoptosis of A549 cells. The PI3K/Akt/mTOR signaling pathway is a key signaling pathway regulating cell proliferation and growth. PI3K is in the upstream of Akt and mTOR. Growth factors can induce the phosphorylation of Akt and mTOR into p-Akt and p-mTOR through activating the PI3K signaling pathway, thereby regulating cell proliferation. According to relevant research, AS, through inhibiting the PI3K/Akt/mTOR signaling pathway, enhances DNA damage repair, induces apoptosis of liver cancer cells, arrests the cell cycle in growth $2 /$ mitotic (G2/M) phase and raises the cell micronucleus rate, thereby enhancing the sensitivity of liver cancer cells to radiotherapy ${ }^{[21]}$. In the present study, the expressions of PI3K, Akt, mTOR, p-PI3K, p-Akt and p-mTOR were detected by western blotting. The results revealed that after treatment with AS, the expressions of PI3K, Akt and mTOR in A549 cells had no obvious changes, while those of p-PI3K, p-Akt and p-mTOR significantly declined, suggesting that the regulatory effects of AS on the proliferation, invasion, migration and apoptosis of A549 cells may be mediated by the PI3K/Akt/mTOR signaling pathway. In conclusion, AS can inhibit the proliferation, invasion and migration, and promote the apoptosis of NSCLC A549 cells in a concentrationdependent manner. The regulatory effects of AS on A549 cell proliferation, migration, invasion and apoptosis may be achieved by inhibiting the expressions of p-PI3K, p-Akt and p-mTOR in the $\mathrm{PI} 3 \mathrm{~K} / \mathrm{Akt} / \mathrm{mTOR}$ signaling pathway, which may provide a new theoretical basis and a potential target for the treatment of NSCLC. In the future, the downstream mechanism of AS on the Akt signaling pathway will be further verified and the potential pathway of AS in the treatment of NSCLC will also be explored.

\section{Acknowledgments:}

This study was financially supported by Qingdao Medical Research Guidance Plan (No. 2019WJZD043).

\section{Conflict of interests:}

The authors declared no conflicts of interest. 


\section{REFERENCES}

1. Public Health Agency of Canada; Statistics Canada; Canadian Cancer Society; provincial/territorial cancer registries. Release notice-Canadian Cancer Statistics 2019. Health Promot Chronic Dis Prev Can 2019;39:255.

2. DeSantis CE, Miller KD, Goding Sauer A, Jemal A, Siegel RL. Cancer statistics for African Americans, 2019. CA Cancer J Clin. 2019;69(3):211-33.

3. Reck M, Rabe KF. Precision diagnosis and treatment for advanced non-small-cell lung cancer. New Eng J Med 2017;377(9):849-61.

4. Herbst RS, Heymach JV, Lippman SM. Lung cancer. N Engl J Med 2008;359:1367-80.

5. Ding L, Getz G, Wheeler DA, Mardis ER, McLellan MD, Cibulskis $\mathrm{K}$, et al. Somatic mutations affect key pathways in lung adenocarcinoma. Nature 2008;455(7216):1069-75.

6. Dhanasekaran SM, Balbin OA, Chen G, Nadal E, KalyanaSundaram S, Pan J, et al. Transcriptome meta-analysis of lung cancer reveals recurrent aberrations in NRG1 and Hippo pathway genes. Nat Commun 2014;5(1):1-2.

7. Wang S, Gao A, Liu J, Sun Y. First-line therapy for advanced non-small cell lung cancer with activating EGFR mutation: is combined EGFR-TKIs and chemotherapy a better choice? Cancer Chemother Pharmacol 2018;81(3):443-53.

8. De Giglio A, Mezquita L, Auclin E, Blanc-Durand F, Riudavets $\mathrm{M}$, Caramella $\mathrm{C}$, et al. Impact of intercurrent introduction of steroids on clinical outcomes in advanced non-small-cell lung cancer (NSCLC) patients under immune-checkpoint inhibitors (ICI). Cancers 2020;12(10):2827.

9. Guibert $\mathrm{N}, \mathrm{Hu}$ Y, Feeney N, Kuang Y, Plagnol V, Jones $\mathrm{G}$, et al. Amplicon-based next-generation sequencing of plasma cell-free DNA for detection of driver and resistance mutations in advanced non-small cell lung cancer. Ann Oncol 2018;29(4):1049-55.

10. Cao B, Dong S, Su Q, Yan B, Zhen H, Yuan Z, et al. Lack of association between the unique LMP2 gene polymorphism and the outcome of lung cancer in a population of Chinese Han nationality. Hum Immunol 2012;73(5):580-4.

11. Li L, Hou X, Xu R, Liu C, Tu M. Research review on the pharmacological effects of astragaloside IV. Fundam Clin Pharmacol 2017;31(1):17-36.

12. Dogar O, Keding A, Gabe R, Marshall AM, Huque R, Barua $\mathrm{D}$, et al. Cytisine for smoking cessation in patients with tuberculosis: a multicentre, randomised, double-blind, placebocontrolled phase 3 trial. Lancet Glob Health 2020;8(11):e140817.

13. Chan WS, Durairajan SS, Lu JH, Wang Y, Xie LX, Kum WF, et al. Neuroprotective effects of Astragaloside IV in 6-hydroxydopamine-treated primary nigral cell culture. Neurochem Int 2009;55(6):414-22.

14. Wang HL, Yang JH. Clinical application and research progress of huisheng oral solution in the treatment of lung cancer. World Latest Med Inf 2019;19:102-3.

15. Zhang A, Zheng Y, Que Z, Zhang L, Lin S, Le V, et al. Astragaloside IV inhibits progression of lung cancer by mediating immune function of Tregs and CTLs by interfering with IDO. J Cancer Res Clin Oncol 2014;140(11):1883-90.

16. Qi H, Wei L, Han Y, Zhang Q, Lau AS, Rong J. Proteomic characterization of the cellular response to chemopreventive triterpenoid astragaloside IV in human hepatocellular carcinoma cell line HepG2. Int J Oncol 2010;36(3):725-35.

17. Garcia J, Hurwitz HI, Sandler AB, Miles D, Coleman RL, Deurloo R, et al. Bevacizumab (Avastin ${ }^{\circledR}$ ) in cancer treatment: A review of 15 years of clinical experience and future outlook. Cancer Treat Rev 2020;86:102017.

18. Hao G, Wang ZG, Fu WY, Yang Y. Research progress on effect of coumarins compounds in anti-tumor. Zhongguo Zhong Yao Za Zhi 2008;33(18):2016-9.

19. Jiang K, Lu Q, Li Q, Ji Y, Chen W, Xue X. Astragaloside IV inhibits breast cancer cell invasion by suppressing Vav3 mediated Rac1/MAPK signaling. Int Immunopharmacol 2017;42:195-202.

20. Zhang XQ, Yao C, Bian WH, Chen X, Xue JX, Zhu ZY, et al. Effects of Astragaloside IV on treatment of breast cancer cells execute possibly through regulation of $\mathrm{Nrf} 2$ via $\mathrm{PI} 3 \mathrm{~K} / \mathrm{AKT} /$ mTOR signaling pathway. Food Sci Nutr 2019;7(11):3403-13.

21. Weinberg MA. RES-529: a PI3K/AKT/mTOR pathway inhibitor that dissociates the mTORC1 and mTORC2 complexes. Anticancer Drugs 2016;27(6):475. 